Bull. Korean Math. Soc. 49 (2012), No. 2, pp. 285-293

http://dx.doi.org/10.4134/BKMS.2012.49.2.285

\title{
HYPERSURFACES WITH CONSTANT $k$-TH MEAN CURVATURE AND TWO DISTINCT PRINCIPAL CURVATURES IN SPHERES
}

\author{
JiANCHENG LiU AND YAN WEI
}

\begin{abstract}
In this paper, we investigate the hypersurface $M$ in a unit sphere with constant $k$-th mean curvature and two distinct principal curvatures, and characterize such a hypersurface.
\end{abstract}

\section{Introduction and main result}

Let $M$ be an $n$-dimensional hypersurface in an $(n+1)$-dimensional unit sphere $S^{n+1}(1)$. It is well known that a compact minimal hypersurface $M$ with $S=n$ in $S^{n+1}(1)$ is isometric to a Clifford torus $S^{1}\left(\sqrt{\frac{1}{n}}\right) \times S^{n-1}\left(\sqrt{\frac{n-1}{n}}\right)$, where $S$ is the squared norm of the second fundamental form of the hypersurface (cf. [5], [7], [8]). In 1970, Otsuki [10] investigated the converse problem by using differential equation and proved that Riemannian product $S^{k}\left(\sqrt{\frac{k}{n}}\right) \times$ $S^{n-k}\left(\sqrt{\frac{n-k}{n}}\right)$ is the only compact minimal hypersurface in $S^{n+1}(1)$ with two distinct principal curvatures whose multiplicities are greater than 1. Furthermore, for compact minimal hypersurfaces with two distinct principal curvatures, one of which is simple, Otsuki also constructed infinitely many immersed minimal hypersurfaces other than the Clifford torus $S^{1}\left(\sqrt{\frac{1}{n}}\right) \times S^{n-1}\left(\sqrt{\frac{n-1}{n}}\right)$ which are not congruent to each other.

In order to characterize the geometric structure of $M$, it is natural to add some additional geometrical or topological conditions on $M$. Based on this consideration, hypersurfaces immersed in $S^{n+1}(1)$ with constant mean curvature or constant scalar curvature have been into our insight and have its own interest. This class of hypersurfaces has been studied by many authors and obtained a series of rigidity or classification results, see [1], [2], [4], [6], [9], [10],

Received October 22, 2010; Revised July 7, 2011.

2010 Mathematics Subject Classification. Primary 53C20.

Key words and phrases. sphere, hypersurface, $k$-th mean curvature, principal curvatures, Riemannian product space.

This work was financially supported by NNSF of China 10871138. 
[11], [12] and the references therein. For example, Wei [11] studied the hypersurfaces in $S^{n+1}(1)$ with constant mean curvature and two distinct principal curvatures and proved that:

Theorem A (Wei [11]). Let $M$ be an $n$-dimensional $(n \geq 3)$ connected complete hypersurface in $S^{n+1}(1)$ with constant mean curvature $H$ and two distinct principal curvatures, one of which is simple. If the squared norm of the second fundamental form $S$ of $M$ satisfies

$$
S \leq n+\frac{n^{3} H^{2}}{2(n-1)}-\frac{n(n-2)}{2(n-1)} \sqrt{n^{2} H^{4}+4(n-1) H^{2}}
$$

or

$$
S \geq n+\frac{n^{3} H^{2}}{2(n-1)}+\frac{n(n-2)}{2(n-1)} \sqrt{n^{2} H^{4}+4(n-1) H^{2}},
$$

then $M$ is isometric to the Riemannian product $S^{1}(a) \times S^{n-1}\left(\sqrt{1-a^{2}}\right)$, where $a^{2}=\frac{2+n H^{2} \pm \sqrt{n^{2} H^{4}+4(n-1) H^{2}}}{2 n\left(1+H^{2}\right)}$.

Concerning the hypersurfaces in $S^{n+1}(1)$ with constant scalar curvature and two distinct principal curvatures, Wei [12] and Cheng [4] proved that:

Theorem B (Wei [12], Cheng [4]). Let $M$ be an $n$-dimensional $(n \geq 3)$ connected complete hypersurface in $S^{n+1}(1)$ with constant scalar curvature $n(n-1) r\left(r \neq \frac{n-2}{n-1}\right.$ is the normalized scalar curvature of $\left.M\right)$ and two distinct principal curvatures, one of which is simple. If

$$
S \leq(n-1) \frac{n(r-1)+2}{n-2}+\frac{n-2}{n(r-1)+2}
$$

or

$$
S \geq(n-1) \frac{n(r-1)+2}{n-2}+\frac{n-2}{n(r-1)+2},
$$

then $M$ is isometric to the Riemannian product $S^{1}\left(\sqrt{1-a^{2}}\right) \times S^{n-1}(a)$, where $a^{2}=\frac{n-2}{n r}$.

Furthermore, the higher order mean curvature extends naturally the mean curvature and the scalar curvature as its special cases. So, studying the structures of hypersurfaces in $S^{n+1}(1)$ with constant $k$-th mean curvature $H_{k}$ (see the Section 2 for definition) is another important research interest. For instance, in [13], Wei characterized the hypersurfaces with $H_{k}=0$ and obtained:

Theorem C (Wei [13]). Let $M$ be an $n$-dimensional $(n \geq 3)$ connected complete hypersurface in $S^{n+1}(1)$ with constant $k$-th mean curvature $H_{k}=0$ and two distinct principal curvatures, one of which is simple.

(i) If $S \geq \frac{n\left(k^{2}-2 k+n\right)}{k(n-k)}$, then $S=\frac{n\left(k^{2}-2 k+n\right)}{k(n-k)}$, and $M$ is isometric to $S^{1}\left(\sqrt{\frac{k}{n}}\right)$ $\times S^{n-1}\left(\sqrt{\frac{n-k}{n}}\right)$. 
(ii) If $S \leq \frac{n\left(k^{2}-2 k+n\right)}{k(n-k)}$, then $S=\frac{n\left(k^{2}-2 k+n\right)}{k(n-k)}$, and $M$ is isometric to $S^{1}\left(\sqrt{\frac{k}{n}}\right)$ $\times S^{n-1}\left(\sqrt{\frac{n-k}{n}}\right)$.

In this paper, we consider $n$-dimensional hypersurfaces with $k$-th mean curvature $H_{k}=$ const. $>0$ in a sphere $S^{n+1}(c)$ with constant curvature $c$. In fact, we prove the following result.

Theorem 1. Let $M$ be an $n$-dimensional $(n \geq 3)$ connected complete hypersurface in $S^{n+1}(c)$ with constant $k$-th $(1 \leq k<n)$ mean curvature $H_{k}(>0)$ and two distinct principal curvatures, one of which is simple. If the squared norm of the second fundamental form $S$ of $M$ satisfies

$$
S \geq(n-1) t_{0}^{\frac{2}{k}}+c^{2} t_{0}^{-\frac{2}{k}},
$$

or

$$
S \leq(n-1) t_{0}^{\frac{2}{k}}+c^{2} t_{0}^{-\frac{2}{k}},
$$

then $M$ is isometric to the Riemannian product $S^{1}\left(c_{1}\right) \times S^{n-1}\left(c_{2}\right)$, where $c_{1}>0$, $c_{2}>0, \frac{1}{c_{1}}+\frac{1}{c_{2}}=\frac{1}{c}$, and $t_{0}$ is the positive real root of the equation $P_{H_{k}}(t) \equiv$ $c k t^{\frac{k-2}{k}}-(n-k) t+n H_{k}=0(t>0)$.

Remark 1. In Section 3, we will prove in Lemma 4 that the equation $P_{H_{k}}(t)=0$ has actually a unique positive real root.

Remark 2. When $k=1, H_{1}$ is exactly the mean curvature $H$. Let $c=1$, then $P_{H_{1}}(t) \equiv \frac{1}{t}-(n-1) t+n H=0(t>0)$ has one positive real root $t_{0}=\frac{n H+\sqrt{n^{2} H^{2}+4(n-1)}}{2(n-1)}$ and Eq. $(2)$ reduces to

$$
S \leq n+\frac{n^{3} H^{2}}{2(n-1)}-\frac{n(n-2)}{2(n-1)} \sqrt{n^{2} H^{4}+4(n-1) H^{2}} .
$$

Therefore, Theorem 1 contains partially Theorem A as its special case. Meanwhile, Eq.(1) reduces to

$$
S \geq n+\frac{n^{3} H^{2}}{2(n-1)}-\frac{n(n-2)}{2(n-1)} \sqrt{n^{2} H^{4}+4(n-1) H^{2}} .
$$

It is obvious that the lower bound of $S$ in Theorem 1 is less than that in Theorem A, this implies that Theorem 1 improves Theorem A partially.

Remark 3. When $k=2$, we have $H_{2}=r-c>0$. Let $c=1$, then $M$ has constant positive second order mean curvature $H_{2}$ if and only if $M$ has constant scalar curvature $r$ and $r>1$, this implies that $r>1-\frac{2}{n}$, and $r \neq \frac{n-2}{n-1}$. In this case, the equation $P_{H_{2}}(t) \equiv-(n-2) t+n H_{2}+2=0(t>0)$ has a unique positive real root $t_{0}=\frac{n(r-1)+2}{n-2}$. Then, (1), (2) reduce to, respectively,

$$
S \geq(n-1) \frac{n(r-1)+2}{n-2}+\frac{n-2}{n(r-1)+2}
$$


and

$$
S \leq(n-1) \frac{n(r-1)+2}{n-2}+\frac{n-2}{n(r-1)+2} .
$$

We infer that Theorem 1 also contains Theorem B ([12, Theorem 1.3] and [4, Theorem 3.1]) as its special cases.

\section{Preliminaries and lemmas}

Let $M$ be an $n$-dimensional hypersurface in an $(n+1)$-dimensional Euclidean sphere $S^{n+1}(c)$ with constant curvature $c$. We choose a local orthonormal frame field $\left\{e_{1}, \ldots, e_{n+1}\right\}$ in $S^{n+1}(c)$, such that $e_{1}, \ldots, e_{n}$ are tangent to $M, e_{n+1}$ is the unit normal vector field. Let $\left\{\omega_{1}, \ldots, \omega_{n+1}\right\}$ denote the corresponding dual coframe field. Using the same symbols as in [10], then the structure equations and the Gauss equations of $M$ can be written as

$$
\begin{gathered}
d \omega_{i}=\sum_{j} \omega_{i j} \wedge \omega_{j}, \quad \omega_{i j}+\omega_{j i}=0, \\
d \omega_{i j}=\sum_{k} \omega_{i k} \wedge \omega_{k j}-\frac{1}{2} \sum_{k, l} R_{i j k l} \omega_{k} \wedge \omega_{l}, \\
R_{i j k l}=c\left(\delta_{i k} \delta_{j l}-\delta_{i l} \delta_{j k}\right)+\left(h_{i k} h_{j l}-h_{i l} h_{j k}\right),
\end{gathered}
$$

where $h_{i j}$ denotes the components of the second fundamental form of $M$. The covariant derivative $h_{i j k}$ of $h_{i j}$ is defined by

$$
\sum_{k} h_{i j k} \omega_{k}=d h_{i j}+\sum_{k} h_{k j} \omega_{k i}+\sum_{k} h_{i k} \omega_{k j}
$$

then, we obtain the Codazzi equation

$$
h_{i j k}=h_{i k j} .
$$

For $1 \leq k \leq n$, the $k$-th mean curvature $H_{k}$ of $M$ is defined by

$$
\left(\begin{array}{l}
n \\
k
\end{array}\right) H_{k}=\sum_{1 \leq i_{1}<\cdots<i_{k} \leq n} \lambda_{i_{1}} \cdots \lambda_{i_{k}},
$$

where $\left(\begin{array}{l}n \\ k\end{array}\right)=\frac{n !}{k !(n-k) !}, \lambda_{i}(1 \leq i \leq n)$ are the principal curvatures of $M$. In particular, when $k=1, H_{1}=H$ is nothing but the mean curvature of $M$; while $k=2$, a simple calculation by using Gauss equations of $M$ yields $H_{2}=r-c$, where $r$ is the normalized scalar curvature of $M$. So we know that the $k$-th mean curvature $H_{k}$ generalizes the mean curvature and the scalar curvature naturally.

Now, we assume that $M$ is a hypersurface in $S^{n+1}(c)$ with constant $k$-th mean curvature $H_{k}(>0)$ and two distinct principal curvatures $\lambda$ (multiplicity $n-1)$ and $\mu$ (multiplicity 1 ). Choosing a proper frame field $\left\{e_{1}, \ldots, e_{n+1}\right\}$ in $S^{n+1}(c)$ such that $h_{i j}=\lambda_{i} \delta_{i j}$, and taking the convention on the range of indices that $1 \leq i, j, k, \ldots \leq n, 1 \leq a, b, c, \ldots \leq n-1$, then

$$
h_{a b}=\lambda \delta_{a b}, h_{n n}=\mu, h_{a n}=0 .
$$


On the other hand, by virtue of the definition of $H_{k}$, we have from (5) that $\left(\begin{array}{l}n \\ k\end{array}\right) H_{k}=\left(\begin{array}{c}n-1 \\ k\end{array}\right) \lambda^{k}+\left(\begin{array}{c}n-1 \\ k-1\end{array}\right) \lambda^{k-1} \mu$, equivalently,

$$
\lambda^{k-1}((n-k) \lambda+k \mu)=n H_{k} .
$$

Notice our assumption $H_{k}>0,(7)$ implies $\lambda \neq 0$ and

$$
\begin{gathered}
\mu=\frac{n H_{k}-(n-k) \lambda^{k}}{k \lambda^{k-1}}, \\
\lambda-\mu=n \frac{\lambda^{k}-H_{k}}{k \lambda^{k-1}} \neq 0 .
\end{gathered}
$$

By means of the following Lemma 3, together with (3), (4), (6) and (9), making use of the similar methods to [10], we get

$$
\omega_{a n}=\frac{\lambda_{, n}}{\lambda-\mu} \omega_{a}=\frac{k \lambda^{k-1} \lambda_{, n}}{n\left(\lambda^{k}-H_{k}\right)} \omega_{a}=\frac{\mathrm{d}\left\{\log \left|\lambda^{k}-H_{k}\right|^{\frac{1}{n}}\right\}}{\mathrm{d} s} \omega_{a} .
$$

Taking exterior differentiation of (10), we have

$$
\begin{gathered}
\mathrm{d} \omega_{a n}=\left\{-\frac{\mathrm{d}^{2}\left\{\log \left|\lambda^{k}-H_{k}\right|^{\frac{1}{n}}\right\}}{\mathrm{d} s^{2}}+\left[\frac{\mathrm{d}\left\{\log \left|\lambda^{k}-H_{k}\right|^{\frac{1}{n}}\right\}}{\mathrm{d} s}\right]^{2}\right\} \omega_{a} \wedge \mathrm{d} s \\
+\frac{\mathrm{d}\left\{\log \left|\lambda^{k}-H_{k}\right|^{\frac{1}{n}}\right\}}{\mathrm{d} s} \sum_{b=1}^{n-1} \omega_{a b} \wedge \omega_{b} .
\end{gathered}
$$

Alternatively, from (10), structure equations and Gauss equations of $M$, a direct calculation gives

$$
\mathrm{d} \omega_{a n}=\frac{\mathrm{d}\left\{\log \left|\lambda^{k}-H_{k}\right|^{\frac{1}{n}}\right\}}{\mathrm{d} s} \sum_{b=1}^{n-1} \omega_{a b} \wedge \omega_{b}-(\lambda \mu+c) \omega_{a} \wedge \mathrm{d} s .
$$

Comparing (11) with (12), we get the following lemma.

Lemma 2. If $M$ is an $n$-dimensional connected complete hypersurface in $S^{n+1}(c)$ with constant $k$-th mean curvature $H_{k}(>0)$ and two distinct principal curvatures $\lambda$ and $\mu$ with multiplicities $n-1$ and 1 , respectively. Then $M$ is the locus of a family of moving submanifolds $M_{1}^{n-1}(s)$ (where the parameter $s$ is the arc length of the integral curves of $\mu$ ), and $\lambda^{k}, H_{k}$ satisfy the following differential equation of order 2 :

$$
-\frac{d^{2}\left\{\log \left|\lambda^{k}-H_{k}\right|^{\frac{1}{n}}\right\}}{d s^{2}}+\left\{\frac{d\left\{\log \left|\lambda^{k}-H_{k}\right|^{\frac{1}{n}}\right\}}{d s}\right\}^{2}+(\lambda \mu+c)=0 .
$$

Lemma 3 (Otsuki [10]). Let $M$ be a hypersurface in a sphere $S^{n+1}(c)$ such that the multiplicities of the principal curvatures are constants, then the distribution of the space of the principal vectors corresponding to each principal curvature is completely integrable. In particular, if the multiplicity of a principal curvature is greater than 1, then this principal curvature is constant on 
each integral submanifold of the corresponding distribution of the space of the principal vectors.

\section{Proof of the main theorem}

Define a positive function $\bar{w}(s)$ over $s \in(-\infty,+\infty)$ by $\bar{w}(s)=\left|\lambda^{k}-H_{k}\right|^{-\frac{1}{n}}$, from (8) and Lemma 2, we get

$$
\frac{\mathrm{d}^{2} \bar{w}}{\mathrm{~d} s^{2}}+\bar{w} \frac{c k \lambda^{k-2}-(n-k) \lambda^{k}+n H_{k}}{k \lambda^{k-2}}=0 .
$$

In order to prove our main theorem, we will prove, at first, that $\frac{\mathrm{d}^{2} \bar{w}}{\mathrm{~d} s^{2}} \geq 0$ or $\frac{\mathrm{d}^{2} \bar{w}}{\mathrm{~d} s^{2}} \leq 0$ by using the equation (13), then to analysis the monotonicity of the functions $\frac{\mathrm{d} \bar{w}}{\mathrm{~d} s}$ and $\bar{w}(s)$. As a result, we will know that $\bar{w}(s)$ is a constant. According to the results due to Cartan [3], taking similar arguments as in [10], we will complete the proof of the main theorem. Whatever, we prove firstly the following lemmas.

Lemma 4. Let $P_{H_{k}}(t)=c k t^{\frac{k-2}{k}}-(n-k) t+n H_{k}$, where $c>0, t>0$, $1 \leq k<n, n \geq 3$, and $H_{k}=$ const. $>0$. Then $P_{H_{k}}(t)$ has a unique positive real root $t_{0}$. Furthermore,

(1) When $0<t \leq t_{0}$, we have $P_{H_{k}}(t) \geq 0$;

(2) When $t \geq t_{0}$, we have $P_{H_{k}}(t) \leq 0$.

Proof. (i) When $k=1$, we have $\frac{\mathrm{d} P_{H_{1}}(t)}{\mathrm{d} t}=-c t^{-2}-(n-1)<0$, which implies that $P_{H_{1}}(t)$ is a strictly monotone decreasing function. The unique positive solution of $P_{H_{1}}(t)=0$ is $t_{0}=\frac{n H_{1}+\sqrt{n^{2} H_{1}^{2}+4(n-1) c}}{2(n-1)}$, thus $P_{H_{1}}(t) \geq 0$ for $0<t \leq$ $t_{0}$ and $P_{H_{1}}(t) \leq 0$ for $t \geq t_{0}$.

(ii) When $k=2$, by making use of the similar methods to (i), we reach the conclusion.

(iii) When $k \geq 3$, a direct calculation then gives $\frac{\mathrm{d} P_{H_{k}}(t)}{\mathrm{d} t}=c(k-2) t^{-\frac{2}{k}}-(n-$ $k$ ) and $\frac{\mathrm{d}^{2} P_{H_{k}}(t)}{\mathrm{d} t^{2}}=-\frac{2(k-2)}{k} c t^{-\frac{2+k}{k}}<0$, which implies that $\frac{\mathrm{d} P_{H_{k}}(t)}{\mathrm{d} t}$ is a strictly monotone decreasing function of $t$. Put $\frac{\mathrm{d} P_{H_{k}}(t)}{\mathrm{d} t}=0$, we get $t_{1}=\left(\frac{n-k}{c(k-2)}\right)^{-\frac{k}{2}}>$ 0 . Thus, if $0<t<t_{1}$, then $\frac{\mathrm{d} P_{H_{k}}(t)}{\mathrm{d} t}>0$ and $P_{H_{k}}(t)$ is strictly monotone increasing. If $t>t_{1}, \frac{\mathrm{d} P_{H_{k}}(t)}{\mathrm{d} t}<0$ and $P_{H_{k}}(t)$ is strictly monotone decreasing. Furthermore, since $\lim _{t \rightarrow 0^{+}} P_{H_{k}}(t)=n H_{k}>0, \lim _{t \rightarrow+\infty} P_{H_{k}}(t)=-\infty$, from the continuous property of $P_{H_{k}}(t)$, we infer that $P_{H_{k}}(t)$ has a unique positive real root, denoted by $t_{0}$. Finally, we conclude that $P_{H_{k}}(t) \geq 0$ for $0<t \leq t_{0}$ and $P_{H_{k}}(t) \leq 0$ for $t \geq t_{0}$, which completes the proof of Lemma 4 .

Lemma 5. Let $f(t)=\frac{1}{k^{2} t^{\frac{2 k-2}{k}}}\left\{(n-1) k^{2} t^{2}+\left(n H_{k}-(n-k) t\right)^{2}\right\}$ for $t>0$, $H_{k}=$ const. $>0,1 \leq k<n$ and $n \geq 3$, then $f\left(t_{0}\right)=(n-1) t_{0}^{\frac{2}{k}}+c^{2} t_{0}^{-\frac{2}{k}}$, where $t_{0}$ is the positive real root of $P_{H_{k}}(t)=0$. Furthermore, if $t \geq H_{k}, f(t)$ is a 
monotone increasing function; if $0<t \leq H_{k}, f(t)$ is a monotone decreasing function.

Proof. Notice that $P_{H_{k}}\left(t_{0}\right)=c k t_{0}^{\frac{k-2}{k}}-(n-k) t_{0}+n H_{k}=0$, so

$$
\begin{aligned}
f\left(t_{0}\right) & =\frac{1}{k^{2} t_{0}^{\frac{2 k-2}{k}}}\left\{(n-1) k^{2} t_{0}^{2}+\left(\left(c k t_{0}^{\frac{k-2}{k}}-(n-k) t_{0}+n H_{k}\right)-c k t_{0}^{\frac{k-2}{k}}\right)^{2}\right\} \\
& =\frac{1}{k^{2} t_{0}^{\frac{2 k-2}{k}}}\left\{(n-1) k^{2} t_{0}^{2}+\left(-c k t_{0}^{\frac{k-2}{k}}\right)^{2}\right\} \\
& =(n-1) t_{0}^{\frac{2}{k}}+c^{2} t_{0}^{-\frac{2}{k}} .
\end{aligned}
$$

Furthermore, we have

$$
\frac{\mathrm{d} f(t)}{\mathrm{d} t}=\frac{2 t^{\frac{2-3 k}{k}}}{k^{3}}\left\{\left(n^{2}-2 n k+n k^{2}\right) t^{2}+n(k-2)(n-k) H_{k} t+(1-k) n^{2} H_{k}^{2}\right\} .
$$

Putting $g(t) \equiv\left(n^{2}-2 n k+n k^{2}\right) t^{2}+n(k-2)(n-k) H_{k} t+(1-k) n^{2} H_{k}^{2}$, a direct calculation gives that $g\left(H_{k}\right)=0$. We will discuss the monotone property of $f(t)$ for $k=1$ and $k \geq 2$ separately.

(1) When $k=1, g(t)=n(n-1) t\left(t-H_{1}\right)$. Henceforth, if $0<t \leq H_{1}$, then $g(t) \leq 0$ and $\frac{\mathrm{d} f(t)}{\mathrm{d} t} \leq 0$, it follows that $f(t)$ is a decreasing function; if $t \geq H_{1}$, then $g(t) \geq 0$ and $\frac{\mathrm{d} f(t)}{\mathrm{d} t} \geq 0$, this leads to $f(t)$ be an increasing function.

(2) When $k \geq 2$, we infer that $\frac{\mathrm{d} g(t)}{\mathrm{d} t}=2 n\left(k^{2}-2 k+n\right) t+n(k-2)(n-k) H_{k}>0$, so $g(t)$ is strictly monotone increasing and $H_{k}$ is the only zero point of $g(t)$. Hence, if $0<t \leq H_{k}$, then $g(t) \leq 0, \frac{\mathrm{d} f(t)}{\mathrm{d} t} \leq 0$ and $f(t)$ is a decreasing function; if $t \geq H_{k}$, then $g(t) \geq 0, \frac{\mathrm{d} f(t)}{\mathrm{d} t} \geq 0$ and $f(t)$ is an increasing function. This completes the proof of Lemma 5 .

Proof of Theorem 1. Put $t=H_{k}$, then $P_{H_{k}}\left(H_{k}\right)=c k H_{k}^{\frac{k-2}{k}}+k H_{k}>0$, we know $H_{k}<t_{0}$ from the monotone property of $P_{H_{k}}(t)$ (Lemma $4(1)$ ). We also assert that $\lambda^{k}>H_{k}$. In fact, if on the contrary $\lambda^{k}<H_{k}$ (because of $\lambda^{k} \neq H_{k}$ from (9)), then $\lambda^{k}<t_{0}$. Review the process of the proof of Lemma 4, it is not difficult to find that $P_{H_{k}}\left(\lambda^{k}\right)>0$. Recall the definition of $P_{H_{k}}(t),(13)$ can be rewritten as

$$
\frac{\mathrm{d}^{2} \bar{w}}{\mathrm{~d} s^{2}}+\bar{w} \frac{P_{H_{k}}\left(\lambda^{k}\right)}{k \lambda^{k-2}}=0
$$

therefore $\frac{\mathrm{d}^{2} \bar{w}}{\mathrm{~d} s^{2}}<0$, this implies $\frac{\mathrm{d} \bar{w}}{\mathrm{~d} s}$ is a strictly monotone decreasing function of $t$, and it has at most one zero point for $s \in(-\infty,+\infty)$. If $\frac{\mathrm{d} \bar{w}}{\mathrm{~d} s}$ has no zero point in $(-\infty,+\infty)$, then $\bar{w}(s)$ is a monotone function in $s \in(-\infty,+\infty)$; if $\frac{\mathrm{d} \bar{w}}{\mathrm{~d} s}$ has one zero point $s_{0}$ in $(-\infty,+\infty)$, then $\bar{w}(s)$ is a monotone function both in $\left(-\infty, s_{0}\right]$ and $\left[s_{0},+\infty\right)$. Since $\bar{w}(s)$ is bounded $([13])$, we know that both 
$\lim _{s \rightarrow-\infty} \bar{w}(s)$ and $\lim _{s \rightarrow+\infty} \bar{w}(s)$ exist and we have

$$
\lim _{s \rightarrow-\infty} \frac{\mathrm{d} \bar{w}(s)}{\mathrm{d} s}=\lim _{s \rightarrow+\infty} \frac{\mathrm{d} \bar{w}(s)}{\mathrm{d} s}=0 .
$$

This is impossible because $\frac{\mathrm{d} \bar{w}(s)}{\mathrm{d} s}$ is a strictly monotone decreasing function. Therefore we prove the assertion that $\lambda^{k}>H_{k}$. By the way, keep in mind that $H_{k}<t_{0}$ as we have proved at the beginning of the proof of Theorem 1.

Evaluating the function $f(t)$ (defined in Lemma 5) at $\lambda^{k}$ and using (7), we easily obtain

$$
\begin{aligned}
f\left(\lambda^{k}\right) & =\frac{1}{k^{2} \lambda^{2 k-2}}\left\{(n-1) k^{2} \lambda^{2 k}+\left[n H_{k}-(n-k) \lambda^{k}\right]^{2}\right\} \\
& =(n-1) \lambda^{2}+\frac{1}{k^{2} \lambda^{2 k-2}} k^{2} \lambda^{2 k-2} \mu^{2}=S .
\end{aligned}
$$

Case 1. If the assumption (1) holds in Theorem 1, i.e., $S=f\left(\lambda^{k}\right) \geq f\left(t_{0}\right)$, we know from Lemma 5 that $\lambda^{k} \geq t_{0}$, thus Lemma 4 tells us $P_{H_{k}}\left(\lambda^{k}\right) \leq$ $P_{H_{k}}\left(t_{0}\right)=0$. So we have $\frac{\mathrm{d}^{2} \bar{w}}{\mathrm{~d} s^{2}} \geq 0$ from (14), this means that $\frac{\mathrm{d} \bar{w}}{\mathrm{~d} s}$ is a monotone increasing function of $s$. Therefore, $\bar{w}(s)$ must be monotonic when $s$ tends to infinity. On the other hand, since $\bar{w}(s)$ is bounded (cf. [13]), we find that both $\lim _{s \rightarrow-\infty} \bar{w}(s)$ and $\lim _{s \rightarrow+\infty} \bar{w}(s)$ exist and we have

$$
\lim _{s \rightarrow-\infty} \frac{\mathrm{d} \bar{w}(s)}{\mathrm{d} s}=\lim _{s \rightarrow+\infty} \frac{\mathrm{d} \bar{w}(s)}{\mathrm{d} s}=0 .
$$

By the monotonicity of $\frac{\mathrm{d} \bar{w}(s)}{\mathrm{d} s}$, we see that $\frac{\mathrm{d} \bar{w}}{\mathrm{~d} s} \equiv 0$, thus $\bar{w}(s)$ is a constant. Then, according to $\bar{w}(s)=\left|\lambda^{k}-H_{k}\right|^{-\frac{1}{n}}$ and (8), we infer that $\lambda, \mu$ are constants on $M$. Therefore, we know from the results due to Cartan in [3] that $M$ is an isoparametric hypersurface, it is isometric to the Riemannian product $S^{1}\left(c_{1}\right) \times S^{n-1}\left(c_{2}\right)$, where $c_{1}>0, c_{2}>0, \frac{1}{c_{1}}+\frac{1}{c_{2}}=\frac{1}{c}$.

Case 2. If the assumption (2) holds in Theorem 1, i.e., $S=f\left(\lambda^{k}\right) \leq f\left(t_{0}\right)$, we obtain from Lemma 5 again that $\lambda^{k} \leq t_{0}$, thus $P_{H_{k}}\left(\lambda^{k}\right) \geq 0$ by Lemma 4 . So we have $\frac{\mathrm{d}^{2} \bar{w}}{\mathrm{~d} s^{2}} \leq 0$, this means that $\frac{\mathrm{d} \bar{w}}{\mathrm{~d} s}$ is a monotone increasing function of $s$. By the similar discussion to the Case 1, we know that $\lambda, \mu$ are constants on $M$ and $M$ is an isoparametric hypersurface, it is isometric to the Riemannian product $S^{1}\left(c_{1}\right) \times S^{n-1}\left(c_{2}\right)$. We complete the proof of Theorem 1 .

Acknowledgements. The authors wish to thank the referee for his/her carefully reading of the original manuscript and to express their gratitude for his/her suggestions which improve the paper.

\section{References}

[1] H. Alencar and M. do Carmo, Hypersurfaces with constant mean curvature in spheres, Proc. Amer. Math. Soc. 120 (1994), no. 4, 1223-1229.

[2] J. N. Barbosa, Hypersurfaces of $S^{n+1}$ with two distinct principal curvatures, Glasg. Math. J. 47 (2005), no. 1, 149-153. 
[3] E. Cartan, Familles de surfaces isoparamétriques dans les espaces á courvure constante, Ann. Mat. Pura Appl. 17 (1938), no. 1, 177-191.

[4] Q. M. Cheng, Hypersurfaces in a unit sphere $S^{n+1}(1)$ with constant scalar curvature, J. London Math. Soc. (2) 64 (2001), no. 3, 755-768.

[5] Q. M. Cheng and S. Ishikawa, A characterization of the Clifford torus, Proc. Amer. Math. Soc. 127 (1999), no. 3, 819-828.

[6] S. Y. Cheng and S. T. Yau, Hypersurfaces with constant scalar curvature, Math. Ann. 225 (1977), no. 3, 195-204.

[7] S. S. Chern, M. do Carmo, and S. Kobayashi, Minimal submanifolds of a sphere with second fundamental form of constant length, In Functional Analysis and Related Fields, pp. 59-75, Springer, 1970.

[8] Jr. H. B. Lawson, Local rigidity theorems for minimal hypersurfaces, Ann. of Math. (2) 89 (1969), 167-179.

[9] H. Li, Global rigidity theorems of hypersurfaces, Ark. Mat. 35 (1997), no. 2, 327-351.

[10] T. Otsuki, Minimal hypersurfaces in a Riemannian manifold of constant curvature, Amer. J. Math. 92 (1970), 145-173.

[11] G. Wei, Complete hypersurfaces with constant mean curvature in a unit sphere, Monatsh. Math. 149 (2006), no. 3, 251-258.

[12] _ Rigidity theorems of hypersurfaces with constant scalar curvature in a unit sphere, Acta Math. Sin. (Engl. Ser.) 23 (2007), no. 6, 1075-1082.

[13] Complete hypersurfaces with $H_{k}=0$ in a unit sphere, Differential Geom. Appl. 25 (2007), no. 5, 500-505.

JIANCHENG LIU

College of Mathematics and Information Science

Northwest Normal University

LANZHOU 730070, P. R. CHINA

E-mail address: liujc@nwnu.edu.cn

YAN WEI

College of Mathematics and Information Science

NORTHWEST NORMAL UNIVERSITY

LANZHOU 730070, P. R. China

E-mail address: weiyanchn@163.com 